\title{
Optimal Diversity Performance of Space Time Block Codes in Correlated Distributed MIMO Channels
}

\author{
Shuangfeng Han, Member, IEEE, Jing Wang, Senior Member, IEEE, Victor O.K. Li, Fellow, IEEE, \\ Shidong Zhou, Member, IEEE, and Kyung Park, Member, IEEE
}

\begin{abstract}
This paper investigates optimal transmission of space-time block codes (STBCs) in distributed multiple-input multiple-output (D-MIMO) Rayleigh fading channels. The optimal diversity performance is achieved through transmit power allocation implemented at the receiver based on transmit and receive correlations to minimize the average symbol error rate (SER). Evaluation of SER performance of uncoded STBCs over a generalized distributed antenna (DA) topology is first presented, with exact analytical SER expressions derived for MQAM and MPSK symbols. SER upper bounds are also derived, based on which two criteria for complexity reduced antenna subset selection with sub-optimal power allocation are further proposed, whose performance approaches optimal over correlated D-MIMO channels. Moreover, a novel simplified but close SER approximation scheme is devised to significantly facilitate optimal SER calculation. We continue to thoroughly analyze how the optimal diversity is affected by large scale fading, targeted data rate, antenna correlations and transmit power. Finally, we develop a surprisingly close and useful analogy between open loop STBCs in co-located MIMO and optimal STBCs in D-MIMO with minimum feedback (i.e., $n$ bits for $n$ DAs in Criterion 2 with power allocation scheme 2 which equally allocates power to the selected DAs). Extensive simulation results have been presented to demonstrate the effectiveness of our analysis.
\end{abstract}

Index Terms-Antenna subset selection, distributed MIMO, space-time block codes, symbol error rate.

\section{INTRODUCTION}

D RIVEN by the needs for high quality of service and high data rate in future wireless communications, multiple antennas have been intensively studied in recent years. Apart from the in-depth research on co-located multiple-input multiple-output (C-MIMO), distributed antennas (DAs) also

Manuscript received January 5, 2006; revised August 17, 2006 and December 25, 2006; accepted January 11, 2007. The associate editor coordinating the review of this paper and approving it for publication was H. Jafarkhani. This work is supported in part by National Natural Science Foundation of China (90204001) and ETRI of Korea.

S. Han was with the Electronics Engineering Department of Tsinghua University, Beijing, China. He is now with Samsung Electronics, Suwon 443738, Korea (e-mail: shuangf.han@samsung.com).

J. Wang and S. Zhou are with the Electronics Engineering Department of Tsinghua University, Beijing, China (e-mail: \{wangj, zhousd\}@ tsinghua.edu.cn).

V. O.K. Li is with the Department of Electrical and Electronic Engineering, University of Hong Kong, Pokfulam, Hong Kong, China (email: vli@eee.hku.hk).

K. Park was with the Electronics and Telecommunications Research Institute of Korea. He is currently with the mobile communication standardization team of PANTECH Co., Seoul, Korea (email: park.kyung@pantech.com).

Digital Object Identifier 10.1109/TWC.2008.06011. received considerable attention. Distributed antenna system (DAS) has attracted attention recently since it can counteract large-scale fading (path loss and shadow fading) and improve coverage, link quality and system capacity [11], [12], [13]. Information theoretic results in [1], [2] have further demonstrated the capacity advantage of DAs over C-MIMO. To fully explore the capacity advantages of DAs, the concept of distributed wireless communication system (DWCS) is proposed [3], [4]. Many cellular service providers or system manufacturers are seriously considering replacing legacy cellular systems with DAS and DWCS or adopting the DAs architecture in the future.

The combination of space-time coding and geographically dispersed antennas in DAS or DWCS seems promising, since it takes advantages of macroscopic diversity, transmit/receive diversity and shortened access distance. However, equal power and rate transmission of space-time codes over distributed MIMO (D-MIMO) channels in DAS or DWCS may result in unacceptable performance due to different large scale fading among the DAs. Therefore, transmit power and rate allocation based on some channel state information (CSI) feedback is indispensable. Some attempts have been made to apply spacetime codes to D-MIMO channels. For high spectral efficiency, a spatial multiplexing scheme is proposed in [5]. When high diversity gain is required, space-time block codes (STBCs) [6] can be utilized to effectively counteract the detrimental effects of channel fading, like the work in [15], [16]. When linear dispersion codes [22] are applied to wireless relay networks, the cooperative diversity performance is addressed in [20], [21].

Among the various space-time codes, STBCs are particularly attractive for practical system deployment due to its decoding simplicity. Since downlink data transmission may be the bottleneck in future wireless communications, we focus in this paper on optimal downlink STBCs design in single user D-MIMO channels. A generalized DA topology is considered, i.e., the DAs are randomly grouped into a number of geographically dispersed ports where the DAs are colocated. Transmit and receive antenna correlations are assumed due to the placement of the arrays or the geometry in the practical transmission scenarios [20]. Following [2], consider a circular area with radius $R$ where the mobile terminal (MT) is at the centre and $N(N=S T)$ DAs are evenly grouped into $S$ uniformly distributed antenna ports. Assuming the port 
density $S / \pi R^{2}=\lambda$, the average square access distance is derived to be $(N+N / S) / 2 \pi \lambda$. Obviously, the fully scattered D-MIMO (i.e. $S=N$ ) has the largest channel capacity and C-MIMO (i.e. $S=1$ ) corresponds to the lowest capacity. However, the generalized DA topology seems more practical since deploying fully scattered DAs is generally rather difficult in some scenarios. Also, multiple DAs in each port can be adaptively configured to accommodate various communication scenarios.

Previously proposed optimal STBCs schemes are generally confined to C-MIMO channels, with various transmitter CSI assumptions. Without CSI, equal transmit power allocation will be adopted [6], [28]. With channel covariance [23] or mean [24] feedback, optimal precoders (beamformers) have been pursued to minimize average symbol error rate (SER). An upper bound of the pairwise error probability (PEP) is minimized in [29] based on covariance feedback. The techniques in [23], [24], [29] consider transmit-only correlation and are well suited to downlink situations where an elevated access point transmits to a MT placed in a rich scattering environment. Recently, precoder designs have been proposed to address both transmit and receive correlation scenarios, e.g. the work in [26].

Actually, D-MIMO is the general form of C-MIMO. The above precoders developed for C-MIMO can be extended to D-MIMO. However, nearly perfect knowledge of channel correlations at the transmitter is generally assumed which may not be feasible in practice due to many reasons such as limited feedback rate and feedback quantization errors. In D-MIMO, each MT may be surrounded by many DAs and need to feed back its transmit and receive correlation matrices including as many DAs as possible before its optimal DA subset (with optimal power allocation) is determined. Heavy system overhead is hence incurred. On the contrary, if each MT selects its optimal DA subset and feeds back the transmit power allocation results efficiently, complexity of system implementation will be significantly reduced.

Moreover, optimal STBCs design in D-MIMO indicates optimal utilization of the diversity potential provided by the channel. For a thorough understanding of optimal diversity of D-MIMO with transmit and receive correlations, we have difficulty in directly applying the existing precoder designs to the above generalized DA topology, since most of them assume receive antennas are uncorrelated. Some features of the optimal precoder are derived in [26], from which an explicit interpretation of optimal diversity performance also seems difficult to be obtained. Based on transmit and receive antenna correlations, simple but effective transmit power allocation schemes at receiver side are indeed well motivated, which are expected to reduce feedback overhead and to yield an explicit diversity analysis.

The error-correcting codes developed for single antenna transmission can be applied directly as outer codes in our system and the uncoded SER criterion will still provide a good indicator for the coded bit error rate (BER) as well. Upper bound for BER has been analyzed for serial concatenation of inner STBCs with outer tuobo code [18] or trellis-coded modulation [17]. In this paper we investigate uncoded STBCs over the generalized DA topology and explore

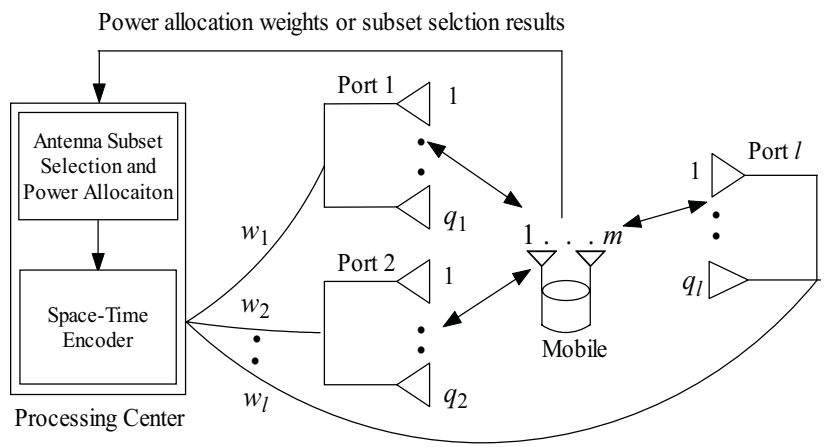

Fig. 1. $\quad\left(n, l, q_{1}, \ldots, q_{l}, m\right)$ D-MIMO channels, where $n$ DAs are grouped into 1 geographically dispersed ports with $q_{j}$ DAs in the $j^{t h}$ port, $m$ mobile antennas are co-located.

efficient receiver-side optimal transmit power allocation based on transmit and receive correlations in flat Rayleigh fading channels to minimize the SER. The main contributions of this paper include:

- For STBCs transmitted over correlated D-MIMO channels with MPSK and MQAM symbols, we derive closedform SER expressions which hold for a generalized DA topology.

- Sub-optimal power allocation schemes with reduced complexity are proposed, which approach the optimal SER performance when combined with antenna subset selection. Tight upper bound on the optimal SER performance is obtained and a surprisingly close and useful analogy is developed between open loop STBC in C-MIMO and optimal STBC in D-MIMO with minimum feedback, facilitating wide applications of STBCs in DAS, DWCS, ad hoc and wireless relay networks.

- Based on extensive SER analysis, the impact of the large scale fading, targeted data rate, antenna correlations and transmit power on the optimal power allocation and diversity order is analyzed.

- A novel scheme is proposed to closely approximate the optimal SER performance of STBCs in correlated DMIMO channels.

The rest of the paper is organized as following. In Sectio II, the system model for D-MIMO is given. In Section III, exact SER expressions for M-QAM and M-PSK symbols are derived. Sub-optimal power allocation schemes are proposed in Section IV, and antenna subset selection criteria are further given. In Section V, numerical results are presented. We conclude this paper in Section VI.

\section{System ModeL}

Consider the downlink single user $\left(n, l, q_{1}, \ldots, q_{l}, m\right)$ DMIMO fading channels. As shown in Fig. 1, $n$ DAs are randomly grouped into $l$ geographically dispersed ports with $q_{j}$ DAs in the $j^{t h}$ port, with $\sum_{j=1}^{l} q_{j}=n$, and the $m$ receive antennas of the mobile are co-located. All the DAs have independent connections to the processing center where transmit and receive signals of DAs are processed. One example of this mechanism can be found in DWCS. Synchronization of transmit signals from different antenna ports is very important, because these signals experience propagation delays that may 
differ by more than a symbol interval. Design of spacetime codes that are robust to differences in the propagation delays can be found in [27]. Generally, the macroscopic and microscopic fading of different DA ports are independent. However, transmit and receive antenna correlations are often observed within each port due to the placement of the array or the geometry in the transmission scenarios.

At the processing center, the optimal transmit antenna subset with the optimal power allocation is first obtained. Then, STBC symbols with unit average power are generated by the space-time encoder and are pre-multiplied before transmission by the power allocation matrix $\mathbf{P}$. We assume the channel is frequency flat and perfect CSI is available at the receiver. The received signal is given as

$$
\mathbf{y}=\sqrt{P_{0}} \mathbf{H} \mathbf{P}^{1 / 2} \mathbf{x}+\mathbf{n}=\sqrt{P_{0}} \mathbf{R}_{r}^{1 / 2} \mathbf{H}_{w} \mathbf{R}_{t}^{1 / 2} \mathbf{P}^{1 / 2} \mathbf{x}+\mathbf{n}
$$

where $\mathbf{y}$ and $\mathbf{x}$ are receive and transmit vectors, $\mathbf{n}$ is an $m \times 1$ noise vector with independent identically distributed (i.i.d.) complex $N(0,1)$ entries and $P_{0}$ is the total average transmit power during one symbol period. $P_{0} / \sigma^{2}$ is the transmit power to receive noise ratio (TSNR) and we denote it by $\rho$. The composite channel matrix $\mathbf{H}$ is expressed as $\mathbf{R}_{r}^{1 / 2} \mathbf{H}_{w} \mathbf{R}_{t}^{1 / 2}$ [7], where $\mathbf{H}_{w}$ is an $m \times n$ matrix with i.i.d. complex $N(0,1)$ entries, $\mathbf{R}_{r}=E\left(\mathbf{H H}^{H}\right) / E\left[\mathbf{H} \mathbf{H}^{H}\right]_{1,1}$ is the normalized $m \times m$ receive correlation matrix with $K$ distinct eigenvalues $\lambda_{i}, i=1, \ldots K$, with $\lambda_{i}$ having algebraic multiplicity $\tau_{i}$, such that $\sum_{i=1}^{K} \tau_{i}=m_{1}$ (i.e. the rank of $\mathbf{R}_{r}$ is $\left.m_{1}\right) \cdot \mathbf{R}_{t}=E\left(\mathbf{H} \mathbf{H}^{H}\right) / m$ is the $n \times n$ transmit correlation matrix with $E\{$.$\} and superscript H$ denoting the expectation and Hermitian transpose of a matrix, respectively. Actually, $\mathbf{R}_{t}=\operatorname{diag}\left(\alpha_{1} \mathbf{R}_{t, 1}, \ldots, \alpha_{l} \mathbf{R}_{t, l}\right)$, where $\mathbf{R}_{t, j}, j=1, \ldots, l$, denotes the $q_{j} \times q_{j}$ normalized (with diagonal entries 1) transmit correlation matrix of the $j^{t h}$ port and $\alpha_{j}$ represents the corresponding large-scale fading. We assume $\mathbf{R}_{t, j}$ has $K_{j}$ distinct eigenvalues $\lambda_{j, k}, k=1, \ldots, K_{j}$, with $\lambda_{j, k}$ having algebraic multiplicity $\tau_{j, k}$ such that $\sum_{k=1}^{K_{j}} \tau_{j, k}=t_{j}$ (i.e. the rank of $\mathbf{R}_{t, j}$ is $t_{j}$ ). Note that $\alpha_{j} \lambda_{j, k}, j=1, \ldots, l, k=1, \ldots, K_{j}$, are eigenvalues of $\mathbf{R}_{t}$. Other non-Kronecker channel correlation models are not considered here.

Without accurate CSI at the transmitter, we assume equal power allocation within each port and denote the power allocation vector by $\mathbf{w}=\left[w_{1}, w_{2}, \ldots, w_{l}\right]$, where $w_{j}, j=1, \ldots, l$, denotes power allocation weight to the $j^{\text {th }}$ port, with $\sum_{j=1}^{l} w_{j}=1$. The diagonal power allocation matrix $\mathbf{P}$ can be expressed as $\mathbf{P}=\operatorname{diag}\left(w_{1} / q_{1}, \ldots, w_{2} / q_{2}, \ldots, w_{l-1} / q_{l-1}, \ldots, w_{l} / q_{l}, \ldots\right)$.

We define transmit antenna subset as any combination of DAs. In total there are $2^{n}-1$ subsets, denoted by $A_{1}, \ldots, A_{2^{n}-1}$. For example, we assume subset $A_{g}$ includes $g$ DA ports with large scale fading $\alpha_{1}, \alpha_{2}, \ldots, \alpha_{g}$ and subset $A_{l}$ includes all the $l$ ports. Denote the spatial rate of STBCs by $r, r=n_{s} / T$, indicating $n_{s}$ independent data streams are transmitted during $T$ consecutive symbol periods. Since different antenna subsets may have different spatial rates, for simplicity, we assume only one spatial rate $r_{k}\left(k=1, \ldots, 2^{n}-1\right)$ for subset $A_{k}$, with the corresponding order of MQAM or MPSK modulations being $M_{k}$.

For a given data rate $R$ and TSNR level $\rho$, the optimal transmit antenna subset and optimal power allocation are obtained at the receiver based on $\mathbf{R}_{t}$ and $\mathbf{R}_{r}$ to minimize the SER. Since $\mathbf{R}_{t}$ and $\mathbf{R}_{r}$ are locally stationary and vary on much slower time scale, the power allocation results can be fed back to the transmitter through a low rate channel. Note that $\mathbf{R}_{t}$ and $\mathbf{R}_{r}$ do not help in transmit power allocation for STBCs in C-MIMO. However, they may be conveniently utilized to counteract the adverse effect of different large scale fading in D-MIMO through optimal transmit power allocation, thus promising a practical system implementation where advantages of both STBCs and DAs are fully exploited.

\section{SER DERIVATION}

The signal to noise ratio (SNR) at detection $\eta$ is a random process due to the randomness of the power of the instantaneous channel realizations. The average SER in the presence of fading can then be obtained by averaging the conditional SER $P(e / x)$ over the distribution of $\eta$, i.e.,

$$
P_{e}=\int_{0}^{\infty} P(e / x) f_{\eta}(x) d x,
$$

where $f_{\eta}(x)$ stands for the probability density function (PDF) of $\eta$. Note that $P(e / x)$ is often represented by the Q-function, $Q(x)=(1 / \sqrt{2 \pi}) \int_{x}^{\infty} \exp \left(-y^{2} / 2\right) d y$, which makes it generally difficult to calculate the integral in (2). With the moment generating function (MGF) of $\eta$ defined as $\Phi_{\eta}(s)=\int_{-\infty}^{\infty} f_{\eta}(x) e^{s x} d x$, alternative SER expressions of coherent M-QAM and M-PSK have been derived as (3) and (4) respectively in [9].

$$
\begin{array}{r}
P_{e, M Q A M}=\frac{4 q}{\pi} \int_{0}^{\pi / 2} \Phi_{\eta}\left(\frac{g_{M Q A M}}{\sin ^{2} \theta}\right) \\
d \theta-\frac{4 q^{2}}{\pi} \int_{0}^{\pi / 4} \Phi_{\eta}\left(\frac{g_{M Q A M}}{\sin ^{2} \theta}\right) d \theta \\
P_{e, M P S K}=\frac{1}{\pi} \int_{0}^{(M-1) \pi / M} \Phi_{\eta}\left(\frac{g_{M P S K}}{\sin ^{2} \theta}\right) d \theta .
\end{array}
$$

with $g_{M Q A M}=1.5 /(M-1), g_{M P S K}=\sin ^{2}(\pi / M)$, and $q=1-1 / \sqrt{M}$. In the following, we will first derive $\Phi_{\eta}(s)$ and then obtain the SER expressions.

STBCs convert the D-MIMO channel into a single-input single-output (SISO) channel with the effective channel gain given by the square-root of the sum of the squared magnitudes of the complex-valued scalar sub-channel gains. Following the procedure outlined in [8], for STBCs transmission over subset $A_{l}$ with power allocation weights $w_{1}, \ldots, w_{l}, \eta$ can be derived to be

$$
\begin{aligned}
\eta & =\left\|\mathbf{R}_{r}^{1 / 2} \mathbf{H}_{w} \mathbf{R}_{t}^{1 / 2} \mathbf{P}^{1 / 2}\right\|_{F}^{2} \rho \\
& =\operatorname{tr}\left(\mathbf{R}_{r}^{1 / 2} \mathbf{H}_{w} \mathbf{R}_{t}^{1 / 2} \mathbf{P} \mathbf{R}_{t}^{\mathbf{H} / 2} \mathbf{H}_{w}^{\mathbf{H}} \mathbf{R}_{r}^{\mathbf{H} / 2}\right) \rho \\
& =\sum_{j=1}^{l} \sum_{k=1}^{K_{j}} \sum_{i=1}^{K}\left(a_{j} w_{j} \rho \lambda_{j, k} \lambda_{i} / q_{j}\right) \sum_{t=1}^{\tau_{j, k} \tau_{i}}\left|h_{t}\right|^{2} \\
& =\sum_{j=1}^{l} \sum_{k=1}^{K_{j}} \sum_{i=1}^{K} \eta_{j, k, i}
\end{aligned}
$$

where $\|\cdot\|_{F}$ denotes the Frobenius norm and $h_{t}, t=$ $1, \ldots, \tau_{j, k} \tau_{i}$ are i.i.d. complex $N(0,1)$ random variables. 
Note that $\eta$ consists of $K \sum_{j=1}^{l} K_{j}$ independent chi-squared random variables $\eta_{j, k, i}$ with $2 \tau_{j, k} \tau_{i}$ degrees of freedom whose MGF is given by $\Phi_{\eta_{j, k, i}}(s)=\left(1-s a_{j} w_{j} \rho \lambda_{j, k} \lambda_{i} / q_{j}\right)^{-\tau_{j, k} \tau_{i}}$. Then, $\Phi_{\eta}(s)$ can be obtained as the product of $\Phi_{\eta_{j, k, i}}(s)$, i.e. $\Phi_{\eta}(s)=\prod_{j=1}^{l} \prod_{k=1}^{K_{j}} \prod_{i=1}^{K} \Phi_{\eta_{j, k, i}}(s)$. Generally, the partial fractions of $\Phi_{\eta}(s)$ are very useful in SER derivation, but the form of the partial fractions depends heavily on the power allocation weights $w_{j}, j=1, \ldots, l$, which have not been determined yet. Without loss of generality, we assume each $a_{j} w_{j}, j=1, \ldots, l$, is different. $\Phi_{\eta}(s)$ can then be resolved into the following partial fractions,

$$
\begin{aligned}
& \Phi_{\eta}(s) \\
& =\prod_{j=1}^{l} \prod_{k=1}^{K_{j}} \prod_{i=1}^{K} \Phi_{\eta_{j, k, i}}(s) \\
& =\prod_{j=1}^{l} \prod_{k=1}^{K_{j}} \prod_{i=1}^{K}\left(1-s a_{j} w_{j} \rho \lambda_{j, k} \lambda_{i} / q_{j}\right)^{-\tau_{j, k} \tau_{i}} \\
& =\sum_{j=1}^{l} \sum_{k=1}^{K_{j}} \sum_{i=1}^{K} \sum_{u=1}^{\tau_{j, k} \tau_{i}} q_{j, k, i, u}\left(1-s a_{j} w_{j} \rho \lambda_{j, k} \lambda_{i} / q_{j}\right)^{-u} \\
& =\sum_{j=1}^{l} \sum_{k=1}^{K_{j}} \sum_{i=1}^{K} \sum_{u=1}^{\tau_{j, k} \tau_{i}} q_{j, k, i, u} \Phi_{\eta_{j, k, i, u}}
\end{aligned}
$$

where $q_{j, k, i, u}$ are obtained by solving a system of linear equations, given as (7) (see next page).

The linearity of the inverse of the MGF allows $f_{\eta}(x)$ to be easily derived to be,

$$
\begin{aligned}
f_{\eta}(x) & =\sum_{j=1}^{l} \sum_{k=1}^{K_{j}} \sum_{i=1}^{K} \sum_{u=1}^{\tau_{j, k} \tau_{i}} \frac{q_{j, k, i, u}}{\Gamma(u)} \\
& \times\left(a_{j} w_{j} \rho \lambda_{j, k} \lambda_{i} / q_{j}\right)^{-u} x^{u-1} e^{-x q_{j} /\left(a_{j} w_{j} \rho \lambda_{j, k} \lambda_{i}\right)}
\end{aligned}
$$

with Gamma function $\Gamma(x)$. Invoking the elegant closedform SER expressions of coherent MQAM and MPSK of STBCs over C-MIMO fading channels given in [10], the SER of STBCs over the $\left(n, l, q_{1}, \ldots, q_{l}, m\right)$ correlated D-MIMO fading channels can be derived as (9) and (10) (see next page), where $F_{1}\left(a, b_{1}, b_{2} ; c, x, y\right)=$ $\sum_{n=0}^{\infty} \sum_{k=0}^{\infty}(a)_{n+k}\left(b_{1}\right)_{n}\left(b_{2}\right)_{k} x^{n} y^{k} /\left((c)_{n+k} n ! k !\right)$ is the Appell Hypergeometric function defined in [23, p.224, Eq. 5.7.(6)], ${ }_{2} F_{1}(a, b ; c, x)=\sum_{n=0}^{\infty}(a)_{n}(b)_{n} x^{n} /\left((c)_{n} n !\right)$ is the Gauss Hypergeometric function defined in $[23$, p.101, Eq. 2.8.(1)] and $(a)_{n}=\Gamma(a+n) / \Gamma(a)$. We observe that the SER expressions are complicated functions of eigenvalues of transmit and receive correlations, large scale fading, TSNR, symbol constellations of STBCs and DA topology. Compared with SER expressions of STBCs in C-MIMO channels, e.g., Eq. 20 and Eq. 25 in [10] or Eq. 39 and Eq. 48 in [30], where all transmit antennas have the same large scale fading and there is no need for power allocation, different large scale fadings are included in (9) and (10). Correspondingly, transmit power shall be optimally allocated to each port to counteract the adverse effect of different large scale fading.

For STBCs transmitted over any other subsets, the corresponding SER expressions can also be obtained. However, the impact of each parameter on SER performance can not be easily understood. In particular, obtaining the optimal power allocation weight $w_{j}$ by minimizing the SER needs complicated computation and it is rather difficult to derive the closed form $w_{j}$. So we resort to bounding techniques to obtain the sub-optimal power allocation and some useful insights into how these parameters affect SER.

\section{Antenna Subset Selection With Sub-Optimal Power Allocation Scheme}

The SER expressions of MQAM ( $M=2^{k}, k$ is even) and MPSK symbols are given in $[14,31]$ as (11) and (12) respectively.

$$
\begin{aligned}
& P_{M Q A M} \\
& =1-\left(1-\left(2-\frac{2}{\sqrt{M}}\right) \int_{0}^{\infty} Q\left(\sqrt{\frac{3 x}{M-1}}\right) f_{\eta}(x) d x\right)_{(11)}^{2} \\
& P_{M P S K}=\int_{0}^{\infty} 2 Q\left(\sqrt{2 x} \sin \frac{\pi}{M}\right) f_{\eta}(x) d x \\
& \quad-\frac{1}{\pi} \int_{0}^{\infty}\left(\int_{\frac{\pi}{2}-\frac{\pi}{M}}^{\frac{\pi}{2}} e^{\left.-x\left(\sin \frac{\pi}{M}\right) / \cos ^{2} \theta d \theta\right) f_{\eta}(x) d x}\right.
\end{aligned}
$$

The SER of BPSK and QPSK over an AWGN channel can be approximated as $P_{B P S K} \approx \int_{0}^{\infty} Q(\sqrt{2 x}) f_{\eta}(x) d x$ and $P_{Q P S K}=\frac{1}{2} \int_{0}^{\infty} Q(\sqrt{x}) f_{\eta}(x) d x$, respectively. For high TSNR level and large values of $M$ (e.g., $M>4$ ), we have $P_{M P S K} \approx \int_{0}^{\infty} 2 Q(\sqrt{2 x} \sin (\pi / M)) f_{\eta}(x) d x$. Alternative SER expressions can be conveniently obtained if we substitute PDF $f_{\eta}(x)$ into (11) and (12) (Note that $\int_{0}^{\infty} Q(\sqrt{a x}) x^{D-1} e^{-x / \beta} d x=$ $\frac{1}{2} \beta^{D} \Gamma(D)\left(1-\sum_{t=0}^{D-1} u\left(\frac{1-\mu^{2}}{4}\right)^{t}\left(\begin{array}{c}2 t \\ t\end{array}\right)\right), \quad \mu \quad=$ $\sqrt{\alpha \beta /(\alpha \beta+2)}$. This can be obtained by the aid of math tools like Mathematica). In the following derivation, we apply bounding technique to the $Q$-function and approximation to $f_{\eta}(x)$ respectively to obtain upper bound and approximation of SER. Since same method can be employed in bounding $P_{M Q A M}$ and $P_{M P S K}$, we take $P_{M Q A M}$ as an example and give the results for MPSK constellations.

\section{A. Chernoff Bound of the Q-function}

Since we do not know the optimal antenna subset for STBCs transmission in a given transmission scenario, without loss of generality, we assume subset $A_{g}$ (with $M_{g}$-QAM symbols) is optimal with optimal power allocation weights $w_{1}, \ldots, w_{g}$, some of which may be zeros. Applying the Chernoff bound to the $Q$ function, i.e., $Q(x) \leq \exp \left(-x^{2} / 2\right)$, SER of $M_{g^{-}}$ QAM constellations can be upper-bounded by (13) (see next page).

Interestingly, the SER upper bound $P_{U B}^{A_{g}}$ is directly related to $\Phi_{\eta}(s)$, making it possible to control SER by adjusting the parameters in $P_{U B}^{A_{g}}$. To minimize $P_{U B}^{A_{g}}$ by optimally allocating transmit power is equivalent to the following optimization problem 


$$
\begin{aligned}
& q_{j, k, i, u}=\frac{-\left(a_{j} w_{j} \rho \lambda_{k, j} \lambda_{i}\right)^{-\left(\tau_{j, k} \tau_{i}-u\right)}}{q_{j}^{-\left(\tau_{j, k} \tau_{i}-u\right)}\left(\tau_{j, k} \tau_{i}-u\right) !} \frac{\partial^{\tau_{j, k} \tau_{i}-u}}{\partial s^{\tau_{j, k} \tau_{i}-u}} \\
& \left(\sum_{\beta=1}^{l} \sum_{\gamma=1}^{K_{j}} \sum_{\substack{\zeta=1 \\
(\beta, \gamma, \zeta) \neq(j, k, i)}}^{K}\left(1-s a_{\beta} w_{\beta} \rho \lambda_{\beta, \gamma} \lambda_{\zeta} / q_{j}\right)^{-\tau_{j, k} \tau_{i}}\right)_{s=\frac{q_{j}}{a_{j} w_{j} \rho \lambda_{j, k} \lambda_{i}}} \\
& P_{e, M Q A M} \\
& =\sum_{j=1}^{l} \sum_{k=1}^{K_{j}} \sum_{i=1}^{K} \sum_{u=1}^{\tau_{j, k} \tau_{i}} q_{j, k, i, u}\left(\begin{array}{c}
\frac{4 q}{\pi} \int_{0}^{\pi / 2} \Phi_{\eta_{j, k, i, u}}\left(-\frac{g_{M Q A M}}{\sin ^{2} \theta}\right) d \theta \\
-\frac{4 q^{2}}{\pi} \int_{0}^{\pi / 4} \Phi_{\eta_{j, k, i, u}}\left(-\frac{g_{M Q A M}}{\sin ^{2} \theta}\right) d \theta
\end{array}\right) \\
& =\sum_{j=1}^{l} \sum_{k=1}^{K_{j}} \sum_{i=1}^{K} \sum_{u=1}^{\tau_{j, k} \tau_{i}} q_{j, k, i, u}\left(\times \frac{\Gamma(u+1 / 2)}{\Gamma(u+1)} F_{2} F_{1}\left(u, 1 / 2 ; u+1 ; \frac{\Phi_{\eta_{j, k, i, u}}\left(-g_{M Q A M}\right) \frac{2 q}{\sqrt{\pi}}}{1+g_{M Q A M} a_{j} w_{j} \rho \lambda_{j, k} \lambda_{i} / q_{j}}\right)\right) \\
& -\sum_{j=1}^{l} \sum_{k=1}^{K_{j}} \sum_{i=1}^{K} \sum_{u=1}^{\tau_{j, k} \tau_{i}} q_{j, k, i, u}\left(\left(\begin{array}{c}
\Phi_{\eta_{j, k, i, u}}\left(-2 g_{M Q A M}\right) \frac{2 q^{2}}{\pi(2 u+1)} \\
\times F_{1}\left(1, u, 1 ; u+3 / 2 ; \frac{q_{j}+g_{M Q A M} a_{j} w_{j} \rho \lambda_{j, k} \lambda_{i}}{q_{j}+2 g_{M Q A M} a_{j} w_{j} \rho \lambda_{j, k} \lambda_{i}}\right), 1 / 2
\end{array}\right)\right) \\
& P_{e, M P S K} \\
& =\sum_{j=1}^{l} \sum_{k=1}^{K_{j}} \sum_{i=1}^{K} \sum_{u=1}^{\tau_{j, k} \tau_{i}} q_{j, k, i, u}\left(\frac{1}{\pi} \int_{0}^{\pi / 2} \Phi_{\eta_{j, k, i, u}}\left(-\frac{g_{M P S K}}{\sin ^{2} \theta}\right) d \theta\right) \\
& +\frac{1}{\pi} \int_{\pi / 2}^{\frac{(M-1) \pi}{M}} \Phi_{\eta_{j, k, i, u}}\left(-\frac{g_{M P S K}}{\sin ^{2} \theta}\right) d \theta \\
& =\sum_{j=1}^{l} \sum_{k=1}^{K_{j}} \sum_{i=1}^{K} \sum_{u=1}^{\tau_{j, k} \tau_{i}} q_{j, k, i, u}\left(\begin{array}{c}
\Phi_{\eta_{j, k, i, u}}\left(-g_{M P S K}\right) \frac{1}{2 \sqrt{\pi}} \frac{\Gamma(u+1 / 2)}{\Gamma(u+1)} \\
\times_{2} F_{1}\left(u, 1 / 2 ; u+1 ; \frac{1}{1+g_{M P S K} a_{j} w_{j} \rho \lambda_{j, k} \lambda_{i} / q_{j}}\right)
\end{array}\right)
\end{aligned}
$$

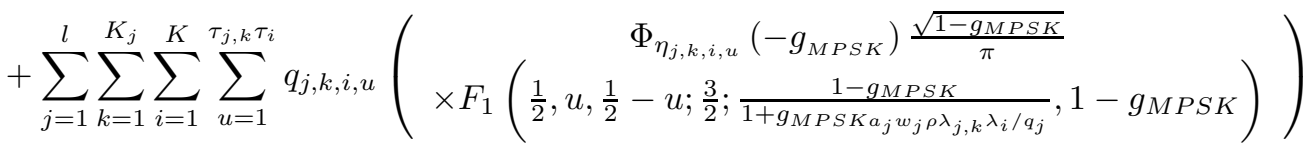

$$
\begin{aligned}
& P_{M_{g}-Q A M}^{A_{g}} \leq 1-\left(1-2\left(1-\frac{1}{\sqrt{M_{g}}}\right) \int_{0}^{\infty} e^{-\frac{3}{2\left(M_{g}-1\right)} x} f_{\eta}(x) d x\right)^{2} \\
& =1-\left(1-2\left(1-\frac{1}{\sqrt{M_{g}}}\right) \Phi_{\eta}\left(-\frac{3}{2\left(M_{g}-1\right)}\right)\right)^{2} \\
& =1-\left(1-2\left(1-\frac{1}{\sqrt{M_{g}}}\right) \prod_{j=1}^{g} \prod_{k=1}^{K_{j}} \prod_{i=1}^{K}\left(1+\frac{3}{2\left(M_{g}-1\right)} a_{j} w_{j} \rho \lambda_{j, k} \lambda_{i} / q_{j}\right)^{-\tau_{j, k} \tau_{i}}\right)^{2} \\
& =P_{U B}^{A_{g}}
\end{aligned}
$$

$$
\begin{aligned}
\mathbf{w}^{*} & =\arg \max \sum_{j=1}^{g} \sum_{k=1}^{K_{j}} \sum_{i=1}^{K} \tau_{j, k} \tau_{i} \\
& \times \log \left(1+\frac{3}{2\left(M_{g}-1\right)} a_{j} w_{j} \rho \lambda_{j, k} \lambda_{i} / q_{j}\right) .
\end{aligned}
$$

We observe the objective for the maximization is concave in the variables $w_{j}$ and can be maximized by the Lagrangian method. Let $g_{j, k}=2 q_{j}\left(M_{g}-1\right) /\left(3 \alpha_{j} \lambda_{j, k} \lambda_{i} \rho\right)$. $w_{j}^{*}, j=$ $1, \ldots, g$, can be easily obtained by solving a set of equations (15) iteratively, and we call this algorithm as Power Allocation
Scheme (PAS) 1.

$$
\frac{\partial F}{\partial w_{j}}=\sum_{k=1}^{K_{j}} \sum_{i=1}^{K} \tau_{j, k} \tau_{i}\left(g_{j, k}+w_{j}\right)^{-1}-u=0
$$

Consequently, the sub-optimal SER performance can be obtained by substituting $w_{j}^{*}$ into (9) or (10). Since the upper bound is not tight, $w_{j}^{*}$ may not be accurate. However, as will be illustrated later by simulations, the sub-optimal SER performance is very close to the optimum.

For a better understanding of the sub-optimal power allocation weights, the following special cases are presented. 


\section{- High TSNR Case}

For sufficiently high TSNR, it is straightforward to show that

$$
w_{j}^{*}=\sum_{k=1}^{K_{j}} \tau_{j, k} / \sum_{j=1}^{g} \sum_{k=1}^{K_{j}} \tau_{j, k},(j=1, \ldots, g) .
$$

Surprisingly, the above simplified sub-optimal power allocation scheme reveals that in high TSNR region the transmit power shall be allocated proportionally over all $g$ antenna ports according to the rank of the transmit correlation matrix of each port, regardless of other parameters in the SER expressions, like TSNR, receive correlations, large scale fading, data rate and spatial rate.

\section{- Fully Correlated Antenna Ports}

When transmit antennas are completely correlated within each port and the receive antennas are also fully correlated, we have $\lambda_{1}=m, \lambda_{i}=0$ for $i=2, \ldots, m$, and $\lambda_{j, 1}=q_{j}$, $\lambda_{j, k}=0$ for $j=1, \ldots, g$ and $k=2, \ldots, q_{j}$. The SER upper bound can be written as

$$
\begin{aligned}
& P_{M_{g}-Q A M}^{A_{g}} \\
& \leq 1-\left(1-\left(2-\frac{2}{\sqrt{M_{g}}}\right) \prod_{j=1}^{g}\left(1+\frac{3 \alpha_{j} w_{j} \rho m}{2\left(M_{g}-1\right)}\right)^{-1}\right)^{2},
\end{aligned}
$$

which is actually a SER upper bound for STBCs over a $(g, g, 1, \ldots, 1,1)$ DA topology with TSNR $\rho m$. In this case, the sub-optimal weights $w_{j}^{*}$ satisfy $w_{j}^{*}=$ $\left(u-2\left(M_{g}-1\right) /\left(3 \alpha_{j} \rho \mathrm{m}\right)\right)_{+}$for $j=1, \ldots, g$, where $u$ is a constant to ensure $\sum_{j=1}^{g} w_{j}^{*}=1$, and $(x)_{+}=x$ (if $x \geq 0$ ) and $(x)_{+}=0$ (if $x<0$ ).

The impact of TSNR, data rate and large scale fading on $w_{j}^{*}$ is thus obvious. For a given data rate and TSNR level, more power will be allocated to the stronger antenna ports. As TSNR increases, the disparity between power allocation weights to the stronger ports and the weaker ports decreases and asymptotically equal power allocation is optimal. At a higher data rate, the disparity between power allocation weights to the stronger ports and the weaker ports increases.

Applying the Chernoff bound to the $Q$ function, SER with $M_{g}$-PSK $\left(M_{g}>4\right)$, BPSK and QPSK symbols can be upperbounded by

$$
\begin{aligned}
& P_{M_{g}-P S K}^{A_{g}} \\
& \leq 2 \prod_{j=1}^{g} \prod_{k=1}^{K_{j}} \prod_{i=1}^{K}\left(1+\sin ^{2}\left(\pi / M_{g}\right) a_{j} w_{j} \rho \lambda_{j, k} \lambda_{i} / q_{j}\right)^{-\tau_{j, k} \tau_{i}} \\
& P_{B P S K}^{A_{g}} \leq \prod_{j=1}^{g} \prod_{k=1}^{K_{j}} \prod_{i=1}^{K}\left(1+a_{j} w_{j} \rho \lambda_{j, k} \lambda_{i} / q_{j}\right)^{-\tau_{j, k} \tau_{i}} \\
& P_{Q P S K}^{A_{g}} \leq 0.5 \prod_{j=1}^{g} \prod_{k=1}^{K_{j}} \prod_{i=1}^{K}\left(1+0.5 a_{j} w_{j} \rho \lambda_{j, k} \lambda_{i} / q_{j}\right)^{-\tau_{j, k} \tau_{i}}
\end{aligned}
$$

Similarly, the sub-optimal power allocation weights for STBCs with $M_{g}$-PSK symbols can also be obtained as (15), with $g_{j, k}=q_{j} /\left(\sin ^{2}\left(\pi / M_{g}\right) a_{j} w_{j} \rho \lambda_{j, k} \lambda_{i}\right), q_{j} /\left(a_{j} w_{j} \rho \lambda_{j, k} \lambda_{i}\right)$, and $2 q_{j} /\left(a_{j} w_{j} \rho \lambda_{j, k} \lambda_{i}\right)$ for $M_{g}$-PSK $\left(M_{g}>4\right)$, BPSK and QPSK constellations, respectively. Note that the power allocation in high TSNR region can also be derived to be (16).

\section{B. Approximation of $f_{\eta}(x)$}

Since the Chernoff bound is not tight, it seems rather difficult to estimate the SER performance directly from the upper bound. In the following, we consider an alternative method, i.e., we first approximate $f_{\eta}(x)$ in (11) and then calculate the integral. Again, without loss of generality, we assume $A_{g}$ is optimal with positive optimal power allocation weights $w_{1}, \ldots, w_{g}$, while $w_{z}=0$, for $z=$ $g+1, \ldots, l$. The characteristic function (CHF) of $\eta$, defined as $\varphi_{\eta}(s)=\int_{0}^{\infty} f_{\eta}(x) e^{I s x} d x$, can be obtained similarly like $\operatorname{MGF} \Phi_{\eta}(s), \varphi_{\eta}(s)=\prod_{j=1}^{g} \prod_{k=1}^{K_{j}} \prod_{i=1}^{K} \varphi_{\eta_{j, k, i}}(s)=$ $\prod_{j=1}^{g} \prod_{k=1}^{K_{j}} \prod_{i=1}^{K}\left(1-s I a_{j} w_{j} \rho \lambda_{j, k} \lambda_{i} / q_{j}\right)^{\tau_{j, k} \tau_{i}}, \quad$ where $\varphi_{\eta_{j, k, i}}(s)$ denotes the CHF of $\eta_{j, k, i}$ and $I=\sqrt{-1}$. Applying the inverse transformation to $\varphi_{n}(s)$, the PDF of $\eta$ can also be expressed as $f_{\eta}(x)=\int_{-\infty}^{+\infty} \varphi_{\eta}(s) e^{-I s x} d s$, which can be further written as (21) (see next page).

Since $A_{g}$ is assumed optimal, contribution of each DA port to the SER performance shall be similar, i.e., similar $\prod_{k=1}^{K_{j}} \prod_{i=1}^{K}\left(1-s I a_{j} w_{j} \rho \lambda_{j, k} \lambda_{i} / q_{j}\right)^{-\tau_{j, k} \tau_{i}}$. Otherwise, some weak DA ports may not be utilized. Denoting $K \sum_{j=1}^{g} K_{j}$ by $d, f_{\eta}(x)$ can be approximated by (22) (see next page). Note that this approximation is close when each $a_{j} w_{j} \rho \lambda_{j, k} \lambda_{i} / q_{j}$ are comparable. Substituting (22) into SER expression with MQAM constellations in (11) and after some mathematical manipulation, the SER when subset $A_{g}$ is assumed optimal is approximated by (23) (see next page), where $C_{1}=\prod_{j=1}^{g} \prod_{k=1}^{K_{j}} \prod_{i=1}^{K}\left(\Gamma\left(\tau_{j, k} \tau_{i}, d\right)\right)^{-\frac{1}{d}}\left(a_{j} w_{j} \rho \lambda_{j, k} \lambda_{i} / q_{j}\right)^{-\tau_{j, k} \tau_{i}}$, $C_{2} \quad=\quad \sum_{j=1}^{g} \sum_{k=1}^{K_{j}} \sum_{i=1}^{K} \frac{q_{j}}{a_{j} w_{j} \rho \lambda_{j, k} \lambda_{i}} \frac{1}{d}, \quad D=$ $\sum_{j=1}^{g} \sum_{k=1}^{K_{j}} \tau_{j, k} \sum_{i=1}^{K} \tau_{i}$ and $\mu=\sqrt{3 /\left(3+2 C_{2}\left(M_{g}-1\right)\right)}$.

In the high TSNR region, $e^{-x C_{2}}$ in $f_{\eta}(x)$ can be further omitted, resulting in a simplified SER expression (24) (see next page) (Note that $\int_{0}^{\infty} Q(\sqrt{a x}) x^{D-1} d x=$ $\left.\alpha^{-D}(2 D)^{-1} \prod_{t=1}^{D}(2 D-(2 t-1))\right)$. The SER approximation and the simplified expression for $M_{g}$-PSK symbols in high TSNR can be similarly derived as (25) (see next page) and (26), respectively.

$P_{M_{g}-P S K}^{A_{g}(H i g h \rho)}=\frac{\prod_{t=1}^{D}(2 D-(2 t-1))}{D}\left(2 \sin ^{2}\left(\pi / M_{g}\right)\right)^{-D} C_{1}$

Actually, we observe from (22) that the approximation is close in high TSNR region. Therefore, $P_{M_{g}-Q A M}^{A_{g}\left(H_{i g h} \rho\right)}$ and $P_{M_{g}-P S K}^{A_{g}\left(H_{i g h} \rho\right)}$ are tight. The sub-optimal power allocation vector $w^{*}$ for subset $A g$ in the high TSNR region can then be obtained by minimizing (24) and (26), which reduces to the 


$$
\begin{aligned}
& f_{\eta}(x)=\int_{-\infty}^{+\infty} \prod_{j=1}^{g} \prod_{k=1}^{K_{j}} \prod_{i=1}^{K}\left(\left(1-s I a_{j} w_{j} \rho \lambda_{j, k} \lambda_{i} / q_{j}\right)^{-\tau_{j, k} \tau_{i} K \sum_{j=1}^{g} K_{j}} e^{-I s x}\right)^{\frac{1}{K \sum_{j=1}^{g} K_{j}}} d s \\
& f_{\eta}(x) \\
& \approx \prod_{j=1}^{g} \prod_{k=1}^{K_{j}} \prod_{i=1}^{K}\left(\int_{-\infty}^{+\infty}\left(1-s I a_{j} w_{j} \rho \lambda_{j, k} \lambda_{i} / q_{j}\right)^{\tau_{j, k} \tau_{i} d} e^{-I s x} d s\right)^{\frac{1}{d}} \\
& =x^{\sum_{j=1}^{g} \sum_{k=1}^{K_{j}} \tau_{j, k} \sum_{i=1}^{K} \tau_{i}-1} \times \prod_{j=1}^{g} \prod_{k=1}^{K_{j}} \prod_{i=1}^{K}\left(\Gamma\left(\tau_{j, k} \tau_{i} d\right)\right)^{-\frac{1}{d}} \\
& \times\left(a_{j} w_{j} \rho \lambda_{j, k} \lambda_{i} / q_{j}\right)^{\tau_{j, k} \tau_{i}} e^{-\frac{q_{j} x}{a_{j} w_{j} \rho \lambda_{j, k} \lambda_{i}} \frac{1}{d}} \\
& =x^{\sum_{j=1}^{g} \sum_{k=1}^{K_{j}} \tau_{j, k} \sum_{i=1}^{K} \tau_{i}-1} e^{-x \sum_{j=1}^{g} \sum_{k=1}^{K_{j}} \tau_{j, k} \sum_{i=1}^{K} \frac{q_{j}}{a_{j} w_{j} \rho \lambda_{j, k} \lambda_{i}} \frac{1}{d}} \\
& \times \prod_{j=1}^{g} \prod_{k=1}^{K_{j}} \prod_{i=1}^{K}\left(\Gamma\left(\tau_{j, k} \tau_{i} d\right)\right)^{-\frac{1}{d}}\left(a_{j} w_{j} \rho \lambda_{j, k} \lambda_{i} / q_{j}\right)^{-\tau_{j, k} \tau_{i}} \\
& P_{M_{g}-Q A M}^{A_{g}} \approx 1-\left(1-C_{1} \Gamma(D)\left(C_{2}\right)^{-D}\left(1-\frac{1}{\sqrt{M_{g}}}\right) \times\left(1-\sum_{t=0}^{D-1} \mu\left(\frac{1-\mu^{2}}{4}\right)^{t}\left(\begin{array}{c}
2 t \\
t
\end{array}\right)\right)\right)^{2}=P_{\text {Approx }}^{A_{g}} \\
& P_{M_{g}-Q A M}^{A_{g}\left(H i g h_{-} \rho\right)}=1-\left(1-\frac{\prod_{t=1}^{D}(2 D-(2 t-1))}{D}\left(1-\frac{1}{\sqrt{M_{g}}}\right)\left(\frac{3}{M_{g}-1}\right)^{-D} C_{1}\right)^{2} \\
& P_{M_{g}-P S K}^{A_{g}} \approx C_{1} \Gamma(D)\left(C_{2}\right)^{-D}\left(1-\sum_{t=0}^{D-1}\left(1+C_{2} / \sin ^{2}\left(\frac{\pi}{M_{g}}\right)\right)^{-0.5} \times\left(\frac{1-\left(1+C_{2} / \sin ^{2}\left(\pi / M_{g}\right)\right)^{-1}}{4}\right)^{t}\left(\begin{array}{c}
2 t \\
t
\end{array}\right)\right)
\end{aligned}
$$

following optimization problem,

$$
w^{*}=\arg \max \prod_{j=1}^{g} \prod_{k=1}^{K_{j}}\left(w_{j}\right)^{\tau_{j, k}}, \text { s.t. } \sum_{j=1}^{g} w_{j}=1
$$

Through the Lagrangian method $w_{j}^{*}$ is derived exactly to be (16), i.e., in high TSNR region transmit power shall be allocated proportionally according to the rank of the transmit correlation matrix of each port. We refer to the scheme which applies (16) at any TSNR level as Power Allocation Scheme 2 (PAS 2). Accordingly, with these sub-optimal weights, the SER performance of each subset can also be obtained from (9) or (10). As will be illustrated later by numerical simulations, PAS 2 also approaches the optimal performance when combined with antenna subset selection, though the power allocation weights are not accurate.

\section{Some Observations}

1) From either (13) or (24) or (26), we observe that the diversity order of this $\left(n, l, q_{1}, \ldots, q_{l}, m\right)$ STBCs is $\sum_{j=1}^{l} \sum_{k=1}^{K_{j}} \tau_{j, k} \sum_{i=1}^{K} \tau_{i}$, which indicates that transmit and receive antenna correlation have similar impact on SER performance, and the case of uncorrelated trans- mit and receive antennas correspond to the best SER performance.

2) Receive correlations have little impact on power allocation in high TSNR region but only degrade the SER performance.

3) Different transmit antenna subsets may have different performance. In the high TSNR region, subset $A_{l}$ performs best because of its highest diversity order. However, for some low or medium TSNR levels, fewer antennas may perform better because antennas with severe large scale fading may waste power. Transmit antenna subset selection with power allocation is indispensable to D-MIMO fading channels.

\section{Transmit Antenna Subset Selection}

For subset $A_{g}$ with spatial rate $r_{g}$ (which corresponds to a certain modulation constellation), PAS 1 can minimize $P_{U B}^{A_{g}}$ by allocating power to each DA port according to (15). Even if some ports of $A_{g}$ are not actually utilized, the modulation constellation remains unchanged. As to PAS 2, $A_{g}$ is assumed optimal and a fixed power allocation (16) is employed. For both schemes, the same problem exists, i.e., is $A_{g}$ the optimal subset out of $A_{1}$ to $A_{2^{n}-1}$ ? 
Given total data rate $R$ and TSNR $\rho$, for optimal transmission of STBCs over correlated D-MIMO channels, we propose the following subset selection criteria with sub-optimal power allocation.

- Criterion 1: Allocate power to antenna subsets $A_{k}$ for $k=1, \ldots, 2^{n}-1$ according to PAS 1 or PAS 2 to obtain the corresponding sub-optimal SER $P_{e}^{A_{k}}$ from SER expressions. The subset with the minimum SER shall be selected for STBCs transmission.

- Criterion 2: Allocate power to antenna subsets $A_{k}$ for $k=1, \ldots, 2^{n}-1$ according to PAS 1 or PAS 2 to obtain the corresponding sub-optimal SER from Chernoff bound based SER upper bounds. The subset with the minimum SER upper bounds shall be selected for STBCs transmission.

\section{E. Some Remarks}

1) The sub-optimal SER performance of STBCs with Criterion 1 over the generalized $\left(n, l, q_{1}, \ldots, q_{l}, m\right)$ DA topology is obtained as

$$
P_{e}=\min \left(P_{e}^{A_{1}}, P_{e}^{A_{2}}, \ldots, P_{e}^{A_{2^{n}-1}}\right) .
$$

Note (28) is a tight upper bound on the optimal SER performance, as shown later by simulations.

2) When subset $A_{g}$ is really optimal, the SER performance of $A_{g}$ can be approximated by (23) or (25). Therefore, it is also a good alternative to substitute the sub-optimal power allocation weights (PAS 2) into SER approximations and select the subset with the best SER for transmission. In scenarios where the approximation is not tight (most often this indicates that subset $A_{g}$ is not optimal), upper bound based method (Criterion 2) can be first utilized to find out the sub-optimal antenna subset and then the SER approximation shall be employed to more accurately approximate the SER performance.

3) Obviously, the complexity of Criterion 1 is much higher than Criterion 2. In the following numerical results, the former only acts as reference, while Criterion 2 is more applicable in real systems.

4) For fully scatted DAs, complexity of the proposed scheme can be significantly reduced by defining antenna subset $A_{g}, g=1, \ldots, n$, as the combination of DAs with large scale fading $\alpha_{1}, \alpha_{2}, \ldots, \alpha_{g}$. In total, only $n$ subsets shall be compared.

5) Since the diversity performance of each port is no worse than that of part of the port, complexity and feedback information of the proposed scheme can also be significantly reduced by only selecting DA ports. When the transmit correlation matrix of each port has full rank, equal power allocation (PAS 2) will be adopted for the selected DA ports. At most $l$ bits feedback information are needed.

6) The proposed framework can be easily extended to Nakagami fading channels. Taking the $(n, n, 1, \ldots, 1, m)$ DA topology with fading parameters $m_{1}, \ldots, m_{n}$ as an example. For antenna subset $A_{n}$ which is assumed to include all $n$ DAs, the sub-optimal power allocation weights can be derived to be $w_{j}=m_{j} / \sum_{j=1}^{n} m_{j}$, $j=1, \ldots, n$.

7) The diversity order of STBCs in D-MIMO fading channels is actually the product of the rank of the transmit correlation matrix corresponding to the selected subset and the rank of the receive correlation matrix, e.g., when subset $A_{g}$ is selected, the diversity order is $\sum_{j=1}^{g} \sum_{k=1}^{K_{j}} \tau_{j, k} \sum_{i=1}^{K} \tau_{i}$. Interestingly, diversity order in D-MIMO practically represents the negative of the slope of SER curve at a given TSNR level and is determined by transmit and receive correlations, large scale fading, spatial rate, TSNR and data rate. For example: 1) The diversity order gets larger as TSNR increases and asymptotically equals the full diversity order $\sum_{j=1}^{l} \sum_{k=1}^{K_{j}} \tau_{j, k} \sum_{i=1}^{K} \tau_{i}$; 2) Given TSNR, the same DA topology may exhibit different diversity order when transmitting different targeted data rates. The higher is the data rate, the lower is the diversity order; 3) The less correlated are the transmit/receive antennas, the higher the diversity order; 4) The more comparable are the large scale fadings of different DA ports, the higher is the diversity order 5) The higher is the spatial rate, the lower is the diversity order.

8) The proposed antenna subset selection with sub-optimal power allocation scheme can also be implemented at the transmitter, with the feedback of eigenvalues of transmit and receiver antenna correlation matrix. However, only port selection is possible and more system overhead is incurred.

9) As will be demonstrated by various numerical results later, the performance of Criterion 2 with PAS 2 is very close to that with PAS 1 . Therefore, the issue of antenna subset selection with sub-optimal power allocation reduces to mere subset selection and $n$ bits are enough for $n$ DAs, because equal power allocation is employed for the selected DAs. A surprisingly close and useful analogy is hence drawn between open loop STBC in C-MIMO and optimal STBC in D-MIMO with minimum feedback. The seemingly adverse effect of different large scale fading in D-MIMO can be readily eliminated by simple antenna selection. This can be extended to cooperative diversity in ad hoc or multi-hop wireless relay systems.

\section{Numerical RESUlts}

For the $\left(n, l, q_{1}, \ldots, q_{l}, m\right)$ D-MIMO channels, we denote the transmit correlation coefficients by $\mathbf{u}=\left[u_{1}, \ldots, u_{l}\right]$, where $u_{j}, j=1, \ldots l$, denotes the correlation coefficient between the adjacent antennas of the $j^{\text {th }}$ port, and we denote the correlation coefficient between the adjacent receive antennas by $v$. The entries of the transmit and receive correlation matrices are given as

$$
\begin{gathered}
\mathbf{R}_{t, j}(p, q)=u_{j}^{|p-q|} \\
\mathbf{R}_{r}(p, q)=\nu^{|p-q|}
\end{gathered}
$$

Note that port selection is assumed in the following numerical results. However, additional simulation results have demon- 


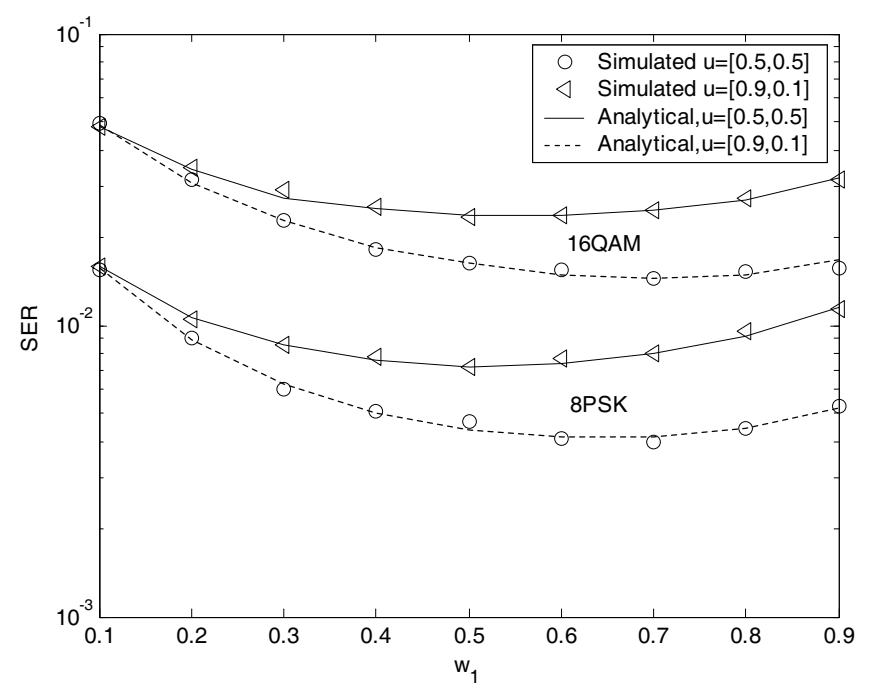

Fig. 2. Simulated and analytical SER vs. $w_{1}$ (power allocation weight to port 1) of STBCs with 16QAM and 8PSK symbols over $(4,2,2,2,2)$ D-MIMO channels where $\mathrm{TSNR}=17 \mathrm{~dB}, \alpha_{1}=1, \alpha_{2}=0.3$ and $v=0.9$.

strated that subset selection from any combination of DAs yields similar results.

\section{A. Verification of SER Expressions in Different Correlation Scenarios}

We first verify the closed form SER expressions in (9) and (10). Consider STBCs over a simple $(4,2,2,2,2)$ D-MIMO channel where 4 DAs are evenly grouped into 2 ports and the receiver has 2 correlated antennas $(v=0.9)$. The normalized large scale fading $\alpha_{1}=1, \alpha_{2}=0.3$ and TSNR is $17 \mathrm{~dB}$. Fig. 2 depicts the SER of STBCs with 16QAM and 8PSK symbols versus $w_{1}$ (power allocation weight to port 1) in 2 correlation scenarios, i.e. $\mathbf{u}=[0.5,0.5]$ and $\mathbf{u}=[0.9,0.1]$. The various lines represent the analytically derived SER, whereas the markers correspond to specific points obtained by means of Monte-Carlo simulations. For all configurations, the simulations clearly corroborate the analytical results.

\section{B. Optimal Power Allocation Weights at Different TSNR Lev- els}

Further simulations of the above DA topology are presented to illustrate the optimal power allocation weights at different TSNR levels. As shown in Fig. 3, SER of STBCs with 16QAM symbols versus $w_{1}$ are simulated at 3 TSNR levels in 2 correlation scenarios, i.e. $\mathbf{u}=[1,0.5]$ and $\mathbf{u}=[0.5,1]$. Fully correlated receive antennas are assumed, i.e. $\nu=1$. We observe that at lower TSNR levels, e.g. 7dB, the SER decreases monotonously as $w_{1}$ increases, indicating the optimality of only port 1 transmitting. As TSNR increases, e.g. to $27 \mathrm{~dB}$, the optimal power allocation weight approaches a fixed value, i.e. $1 / 3$ and $2 / 3$ for the 2 correlation scenarios respectively. This can verify very well the sub-optimal $w_{1}^{*}$ obtained from (16).

\section{Sub-Optimal Power Allocation Weights of PAS 1}

Consider STBCs transmitted over a $(6,3,2,2,2,1)$ DA topology where 6 DAs are equally grouped into 3 ports. We assume

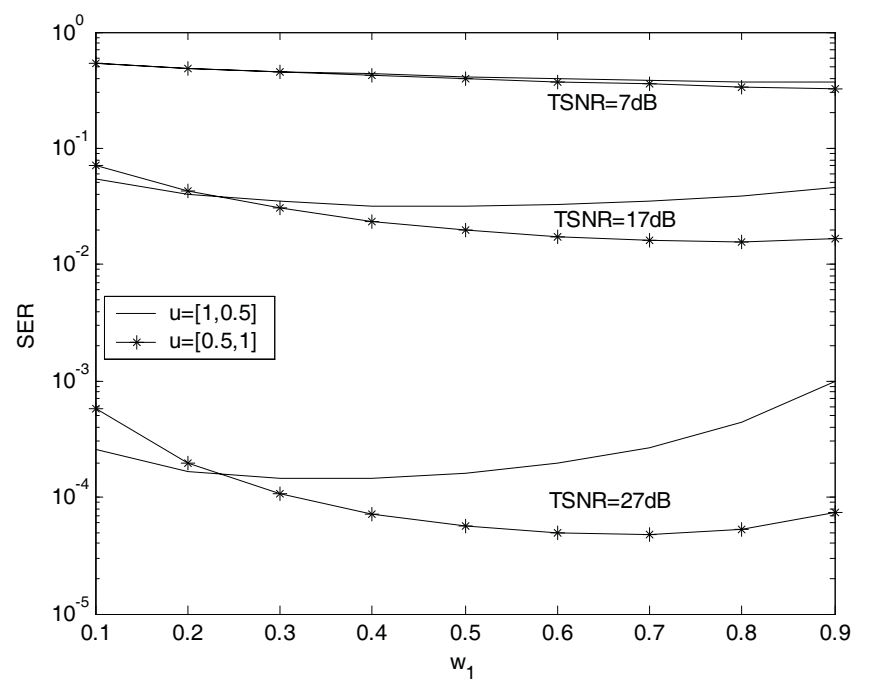

Fig. 3. SER vs. $w_{1}$ (power allocation weight to port 1) of STBCs with 16QAM symbols over $(4,2,2,2,2)$ D-MIMO channels where $v=0.9, \alpha_{1}=1$, $\alpha_{2}=0.3$.
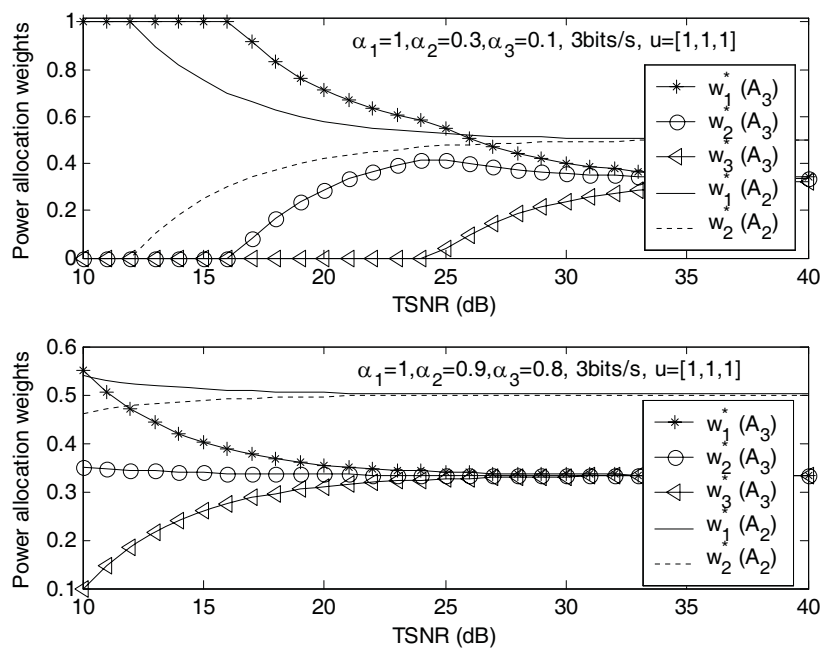

Fig. 4. Power allocation weights vs. TSNR for STBCs over $(6,3,2,2,2,1)$ D-MIMO channels in Scenarios 1 and 2.

antenna subset $A_{1}$ includes port 1 DAs, $A_{2}$ includes port 1 and port 2 DAs, and $A_{3}$ includes the DAs in all 3 ports. Four system scenarios are simulated to illustrate the influence of large-scale fading, data rate and transmit correlations on the sub-optimal power allocation. Scenario 1 is simulated as a baseline, with $\mathbf{u}=[1,1,1]$, 3bits/s data rate and normalized large scale fading $\alpha_{1}=1, \alpha_{2}=0.3$, and $\alpha_{3}=0.1$. For Scenario 2, large scale fading is changed to $\alpha_{1}=1, \alpha_{2}=0.9$, and $\alpha_{3}=0.8$. For Scenario 3, a larger data rate, 6bits/s, is transmitted. Finally, correlation coefficients for Scenario 4 are changed to $\mathbf{u}=[0,1,1]$. For Scenarios 1, 2 and 3, full transmit correlation is assumed within each port and the system configuration is equivalent to a $(3,3,1,1,1,1)$ system. We assume full rate (8PSK symbols for 3bits/s and 64QAM for 6bits/s) for $A_{1}$ and $A_{2}$ and 3/4 rate (16QAM symbols for $3 \mathrm{bits} / \mathrm{s}$ and 256QAM for 6bits/s) for $A_{3}$. While for Scenario 4, full rate for $A_{1}$ and $3 / 4$ rate for $A_{2}$ and $A_{3}$ are assumed. The sub-optimal power allocation weights obtained from (15) are displayed in Fig. 4 and Fig. 5. 

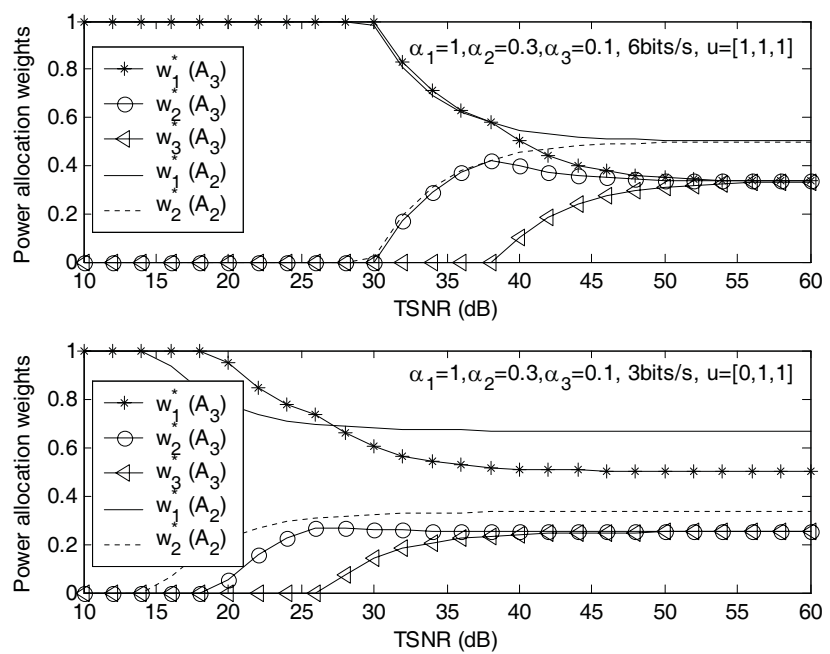

Fig. 5. Power allocation weights vs. TSNR for STBCs over $(6,3,2,2,2,1)$ D-MIMO channels in Scenarios 3 and 4.

As depicted in Fig. 4, for subset $A_{3}$, when TSNR is above $24 \mathrm{~dB}$, DAs in all 3 ports shall be utilized, with more power allocated to port 1 and least power to port 3. As TSNR increases, more balanced power allocation is observed. However, when TSNR is between 16 to $24 \mathrm{~dB}$, only ports 1 and 2 are useful. When TSNR is below $16 \mathrm{~dB}$, only the strongest port 1 DAs shall be selected. As to subset $A_{2}$, when TSNR is above $12 \mathrm{~dB}$, ports 1 and 2 shall be selected and when TSNR is below $12 \mathrm{~dB}$, only the strongest port 1 is selected. Note that $A_{3}$ can actually be employed only when TSNR is beyond $24 \mathrm{~dB}$ and $A_{2}$ can actually be employed when TSNR is beyond $12 \mathrm{~dB}$.

In Scenario 2, all the ports within each antenna subset are utilized in the TSNR region considered. This can be explained by the fact that $\alpha_{1}, \alpha_{2}$, and $\alpha_{3}$ are more comparable than those in Scenario 1. In Scenario 3, we observe from Fig. 5 that port 2 DAs of both $A_{2}$ and $A_{3}$ will not be utilized until TSNR is over $30 \mathrm{~dB}$ and port $3 \mathrm{DAs}$ of $A_{3}$ will be utilized only when TSNR is beyond $38 \mathrm{~dB}$. This indicates that at a given TSNR level less antenna ports will be utilized when a higher data rate is transmitted. In Scenario 4, port 1 DAs are assumed uncorrelated. Consequently, in high TSNR region we observe that $2 / 3$ power will be allocated to port 1 in $A_{2}$ and $1 / 2$ power to port 1 in $A_{3}$.

\section{Comparison of Sub-Optimal Power Allocation Schemes}

Consider the above $(6,3,2,2,2,1)$ STBCs in Scenario 1 . The SER performance of $A_{2}$ and $A_{3}$ are depicted in Fig. 6, where both PAS 1 and PAS 2 (equal power allocation in this case) are adopted. The results are surprisingly satisfactory, i.e., SER performance of $A_{2}$ and $A_{3}$ with PAS 2 is very close to that with PAS 1, especially in the TSNR region where these subsets are actually utilized, e.g., when TSNR is beyond $24 \mathrm{~dB}$ for $A_{3}$ and when TSNR is beyond $12 \mathrm{~dB}$ for $A_{2}$, as shown in Fig. 4.

The Chernoff inequality based upper bounds of $A_{2}$ and $A_{3}$ with PAS 1 and PAS 2 are also depicted, which are not tight for the whole TSNR region plotted. However, we observe SER upper bounds of each subset with PAS 1 are quite close to those with PAS 2. Also, the intersection points of $A_{2}$ and $A_{3}$ with either PAS 1 or PAS 2 are very close to the intersection

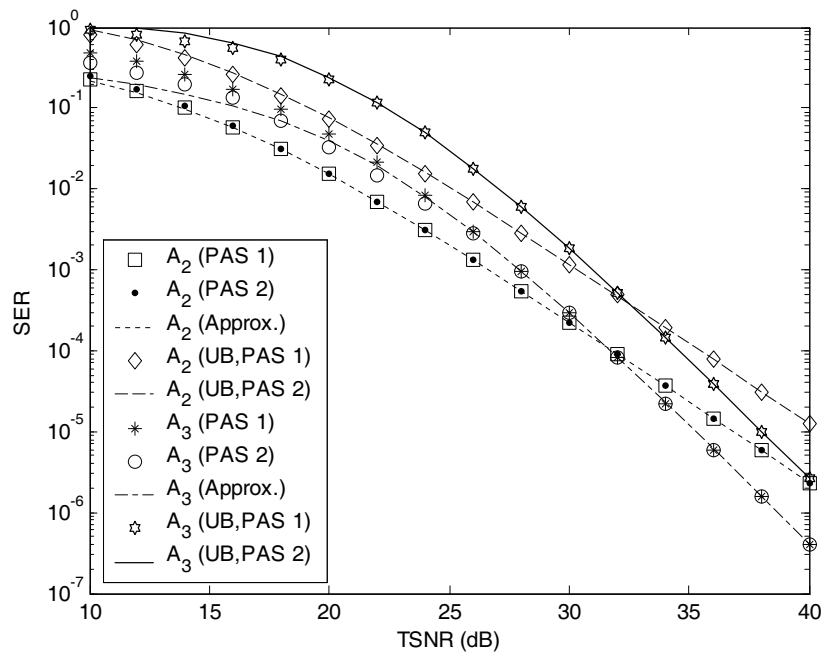

Fig. 6. SER vs. TSNR of antenna subsets $A_{2}$ and $A_{3}$ of STBCs over $(6,3,2,2,2,1)$ D-MIMO channels where $\mathbf{u}=[1,1,1], \alpha_{1}=1, \alpha_{2}=0.3$, and $\alpha_{3}=0.1$. 8PSK symbols for full rate $A_{2}$ and 16QAM symbols for $3 / 4$ rate $A_{3}$.

point of the actual SER curves, indicating applicability of antenna subset selection criterion 2. The SER approximations of $A_{2}$ and $A_{3}$ with PAS 2 are also depicted, which are much tighter than the upper bounds. In the much interested SER range, e.g., $10^{-3}$ to $10^{-2}$, these approximations are very close to the actual SER performance.

\section{E. Antenna Subset Selection with Sub-Optimal Power Alloca- tion}

Antenna subset selection criteria for STBCs transmission over D-MIMO channels are illustrated by the above $(4,2,2,2,2)$ DA topology. The targeted data rate is 3 bits/s. There are 3 antenna subsets, including full rate $A_{1}$ (port 1 DAs) with 8PSK symbols, $3 / 4$ rate $A_{2}$ (ports 1 and 2 DAs) with 16QAM symbols, and full rate $A_{3}$ (port 2 DAs) with 8PSK symbols. For reference, we obtain the optimal SER performance of $A_{2}$ by optimally allocating transmit power between the two ports, and in this process we maintain 16QAM constellations although for some TSNR levels port 2 DAs may not be used. 2 scenarios are studied, including Scenario 1 where $\mathbf{u}=[0.5,1]$, $\alpha_{1}=1, \alpha_{2}=0.3$ and Scenario 2 where $\mathbf{u}=[0.5,1]$, $\alpha_{1}=1$ and $\alpha_{2}=0.01$. Since the SER performance of $A_{3}$ is worse than $A_{1}$, we only consider subsets $A_{1}$ and $A_{2}$ in subset selection. The SER performance of $A_{1}$, SER approximation of $A_{1}$, upper bonds of $A_{1}, A_{2}$ with PAS 1 , $A_{2}$ with PAS $2, A_{2}$ with equal power allocation, $A_{2}$ with optimal power allocation, SER approximation of $A_{2}$ with PAS 2, upper bounds of $A_{2}$ with PAS 1 and PAS 2, $A_{2}$ with optimal power allocation, and SER performance with antenna subset selection are depicted for the 2 scenarios in Fig. 7 and Fig. 8 respectively.

For Scenario 1, we find that the sub-optimal performance of $A_{2}$ with PAS 2 is very close to the performance of $A_{2}$ with PAS 1 and the optimal performance, even in low TSNR region. With antenna subset selection, $A_{1}$ is selected when TSNR is lower than $24 \mathrm{~dB}$ and $A_{2}$ is selected when TSNR is beyond $24 \mathrm{~dB}$. The benefit of antenna subset selection compared with equal power allocation over $A_{2}$ is obvious, e.g. a $2.5 \mathrm{~dB}$ 


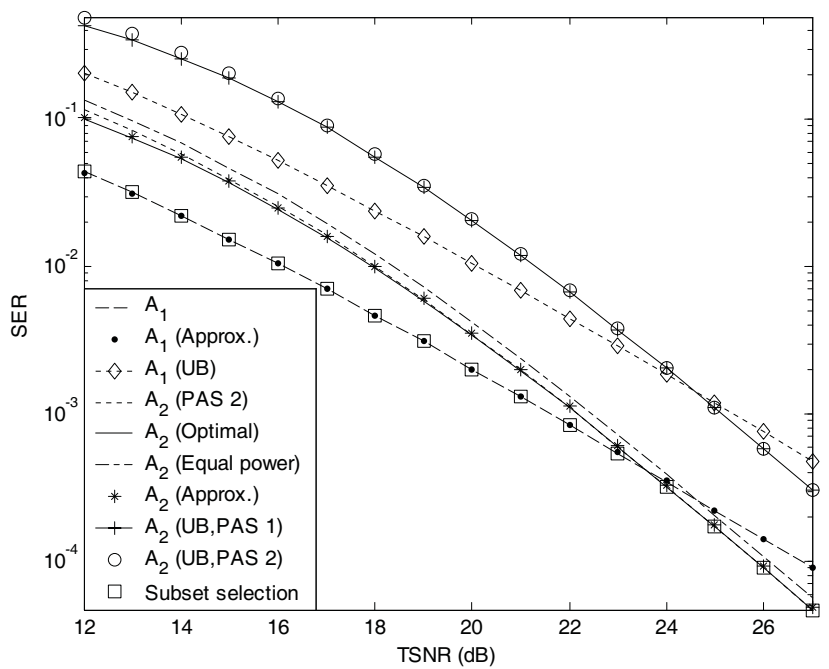

Fig. 7. SER vs. TSNR of antenna subsets $A_{1}$ and $A_{2}$ of STBCs over $(4,2,2,2,2)$ D-MIMO channels where $\mathbf{u}=[0.5,1], v=0.9, \alpha_{1}=1, \alpha_{2}=$ $0.3,8$ PSK symbols for full rate $A_{1}$ and 16QAM symbols for $3 / 4$ rate $A_{2}$.

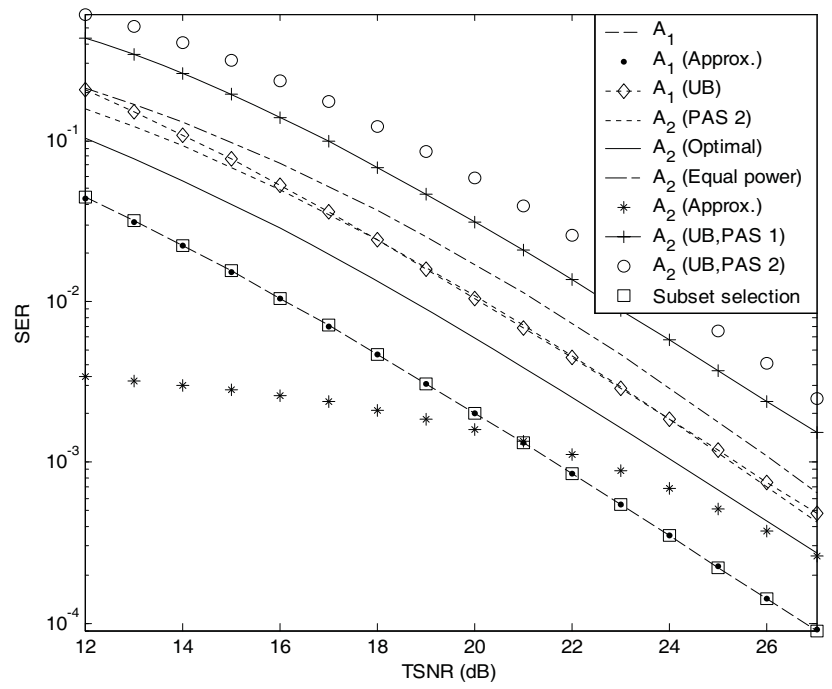

Fig. 8. SER vs. TSNR of antenna subsets $A_{1}$ and $A_{2}$ of STBCs over $(4,2,2,2,2)$ D-MIMO channels where $\mathbf{u}=[0.5,1], v=0.9, \alpha_{1}=1, \alpha_{2}=$ $0.3,8$ PSK symbols for full rate $A_{1}$ and 16QAM symbols for $3 / 4$ rate $A_{2}$.

reduction of TSNR at $10^{-2}$ SER. We also find that SER approximations of $A_{1}$ and $A_{2}$ are very close to the actual SER, ensuring subset selection based on these lower bounds yields exactly the same results, though with much reduced complexity. Finally, we gladly observe that subset selection based on upper bounds of $A_{1}$ and $A_{2}$ (with either PAS 1 or PAS 2) incurs very little performance degradation, e.g., less than $1 \mathrm{~dB}$ in this scenario.

Intuitively, when $\alpha_{1}$ is much larger than $\alpha_{2}, A_{1}$ will outperform $A_{2}$ in a much broader TSNR region. For Scenario 2 , the SER performance of $A_{1}$ is found to outperform $A_{2}$ in the whole plotted 12 to $27 \mathrm{~dB}$ TSNR region. A more significant performance improvement of subset selection over equal power transmission is observed, e.g. 5.6dB TSNR reduction at $10^{-2}$ SER promised by antenna subset selection. Similar to Scenario 1, upper bound based subset selection also yields very good performance. However, SER approximation based subset selection does not work, because the SER approxi- mation of $A_{2}$ with PAS 2 is quite loose at lower TSNR region, which may be explained by the large gap between $\alpha_{1}$ and $\alpha_{2}$. However, our SER approximation of optimal antenna subset should not be questioned, because subset $A_{2}$ is not optimal in this scenario. Therefore, the application of SER approximation based subset selection shall be combined with upper bound based method, i.e., given a TSNR level, upper bound based method is applied first to decide the sub-optimal antenna subset, then SER approximation of the selected subset can be utilized to more accurately approximate the actual SER.

For the above 2 scenarios, we observe that the upper bound on the optimal SER performance in (28) corresponds to the SER curves with antenna subset selection in Fig. 7 and Fig. 8 , indicating the tightness of this upper bound.

Also note that the diversity order of $A_{1}$ and $A_{2}$ in the high power region is 2 and 3 respectively. But the diversity is 2 under $24 \mathrm{~dB}$ and 3 beyond $24 \mathrm{~dB}$ in Scenario 1, indicating more transmit antennas are selected as TSNR increases.

\section{CONCLUSIONS}

This paper investigates optimal diversity performance of STBCs in D-MIMO channels. Transmit power allocation is implemented at the receiver based on the second order channel statistics to minimize the SER. For STBCs with MQAM and MPSK symbols transmitted over a generalized antenna topology in flat Rayleigh fading channels with transmit and receive antenna correlations, the closed-form SER expressions are derived. Upper bounds on SER are also derived, based on which sub-optimal power allocation schemes with reduced complexity are further proposed, including 'water pouring' scheme PAS 1 and fixed power allocation scheme PAS 2. PAS 2 allocates transmit power proportionally over antenna ports according to the rank of the transmit correlation matrix of each port, regardless of other parameters in the SER expressions.

Efficient antenna subset selection criteria are further proposed, which adopts either PAS 1 or PAS 2 and selects the subset with the minimum SER or SER upper bound for transmission. Numerical results have demonstrated that transmission of STBCs with the proposed criteria achieve the optimal diversity performance over correlated D-MIMO channels. In particular, Criterion 2 with PAS 2 is more appealing, which reduces the optimal transmit power allocation issue to antenna subset selection by equally allocating power to the selected DAs, thus significantly reducing feedback overhead, e.g., at most $n$ bits for n DAs. Actually, STBCs with Criterion 2 (PAS 2) in D-MIMO channels are analogous to open loop STBCs in CMIMO. With inherent macroscopic diversity, transmit/receive diversity and shortened access distance, STBCs with Criterion 2 (PAS 2) may have wider applications in future wireless communications. Combined with Criterion 2, a close SER approximation is also presented, which can be directly utilized for performance prediction of STBCs in D-MIMO channels.

\section{ACKNOWLEDGEMENT}

The authors wish to thank the Associate Editor Prof. Hamid Jafarkhani for detailed suggestions and insightful comments that greatly improved the presentation of this paper. The authors would also like to thank the anonymous reviewers for their careful reading and valuable critique of this manuscript. 


\section{REFERENCES}

[1] W. Roh and A. Paulraj, "MIMO channel capacity for the distributed antenna systems," in Proc. IEEE VTC, 2002, vol. 2, pp. 706-709.

[2] H. Zhuang, L. Dai, L. Xiao and Y. Yao, "Spectral efficiency of distributed antenna system with random antenna layout," IEEE Electron. Lett., vol. 39, no. 6, pp. 495-496, 2003.

[3] S. Zhou, M. Zhao, X. Xu, J. Wang, and Y. Yao, "Distributed wireless communication system: A new architecture for future public wireless access," IEEE Commun. Mag., vol. 41, no. 3, pp 108-113, 2003.

[4] S. Zhou, Y. Li, M. Zhao, X. Xu, J. Wang, and Y. Yao, "Novel techniques to improve downlink multiple access capacity for Beyond 3G," IEEE Commun. Mag., vol. 41, no. 3, pp. 61-69, 2005.

[5] S. Han, S. Zhou, J. Wang, and W. Park, "Transmit antenna selection with power and rate allocation for spatial multiplexing in distributed antenna systems," Tsinghua Science Technol., vol. 11, no. 3, pp. 259-263, June 2006.

[6] V. Tarokh, H. Jafarkhani, and A. R. Calderbank, "Space-time block codes from orthogonal designs," IEEE Trans. Inform. Theory, vol. 45, pp. 1456-1467, July 1999.

[7] C. N. Chuah, D. N. C. Tse, and J. M. Kahn, "Capacity scaling in MIMO wireless systems under correlated fading," IEEE Trans. Inform. Theory, vol. 48, pp. 637-650, Mar. 2002.

[8] E. G. Larsson and P. Stoica, Space-Time Block Coding for Wireless Communications. Cambridge, UK: Cambridge Univ. Press, 2003.

[9] M. O. Hasn and M. S. Alouini, "Performance analysis of two-hop relayed transmissions over Rayleigh fading channels," in Proc. IEEE VTC, 2002, vol. 4, pp. 1992-1996.

[10] H. Shin and J. H. Lee, "Exact symbol error probability of orthogonal space-time block codes," in Proc. IEEE Globecom, Nov. 2002, pp. 1547 1552.

[11] A. Saleh, A. Rustako, and R. Roman, "Distributed antennas for indoor radio communication," IEEE Trans. Commun., vol. 35 no. 12, pp. 12451251, Dec. 1987.

[12] W. Roh, and A. Paulraj, "Outage performance of the distributed antenna systems in a composite fading channel," in Proc. IEEE VTC, vol. 3, pp. 1520-1524.

[13] P. Chow, A. Karim, V. Fung, and C. Dietrich, "Performance advantages of distributed antennas in indoor wireless communication systems," in Proc. IEEE VTC, 1994, vol. 3, pp. 1522-1526.

[14] J. G. Proakis, Digital Communications, 3rd ed. New York: McGrawHill, 1995

[15] M. Dohler, "Virtual antenna arrays," PhD thesis, University of London, 2003.

[16] S. Han, J. Wang, V. O. K. Li, S. Zhou, and K. Park, "Sub-optimal transmission of orthogonal space time block codes over correlated distributed antennas," IEEE Signal Processing Lett., vol. 14, no. 2, pp. 89-92, Feb. 2007.

[17] M. Uysal and C. N. Georghiades, "Upper bounds on the BER performance of MTCM-STBC schemes over shadowed Rician fading channels," Eurasip J. Applied Signal Processing, Special Issue Advances Smart Antennas," vol. 2004, no. 9, pp. 1238-1245, Aug. 2004.

[18] J. Hu and S. L. Miller, "Performance analysis of convolutionally coded systems over quasi-static fading channels," IEEE Trans. Wireless Commun., vol. 5, no. 4, pp. 789-795, Apr.. 2006.

[19] D. Shiu, G. J. Foschini, M. J. Gans, and J. M. Kahn, "Fading correlation and its effect on the capacity of multielement antenna systems," IEEE Trans. Commun., vol. 48, no. 3, pp. 502-513, Mar. 2000.

[20] Y. Jing and B. Hassibi, "Distributed space-time coding in wireless relay networks-Part I: Basic diversity results," to be published.

[21] Y. Jing and B. Hassibi, "Distributed space-time coding in wireless relay networks-Part II: Tighter upper bounds and a more general case," to be published.

[22] B. Hassibi and B. Hochwald, "High-rate codes that are linear in space and time," IEEE Trans. Inform. Theory, vol. 48, pp. 1804-1824, July 2002.

[23] A. Erdelyi, Higher Transcendental Functions, vol. 1. New York: McGraw-Hill, 1953.

[24] S. Zhou and G. B. Giannakis, "Optimal transmitter eigen-beamforming and space-time block coding based on channel correlations," IEEE Trans. Inform. Theory, vol. 49, no. 7, pp. 1673-1690, July 2003.

[25] S. Zhou, and G. B. Giannakis, "Optimal transmitter eigen-beamforming and space-time block coding based on channel mean feedback," IEEE Trans. Signal Processing, vol. 50, no. 10, pp. 2599-2613, Oct. 2002.

[26] A. Hjorungnes and D. Gesbert, "Precoding of orthogonal space-time block codes in arbitrarily correlated MIMO channels: Iterative and closed-form solutions," to be published.
[27] D. Goeckel and Y. Hao, "Macroscopic space-time coding: motivation, performance criteria, and a class of orthogonal designs," in Proc Conference Inform. Sciences Syst., Mar. 2000.

[28] G. Ganesan and P. Stoica, "Space-time block codes: A maximum SNR approach," IEEE Trans. Inform. Theory, vol. 47, pp. 1650-1656, May 2001.

[29] H. Sampath and A. Paulraj, "Linear precoding for space-time coded systems with known fading correlations," IEEE Commun. Lett., vol. 6, no. 6, pp. 239-241, June 2002 .

[30] H. Zhang and T. A. Gulliver, "Capacity and error probability analysis for orthogonal space-time block codes over fading channels," IEEE Trans. Wireless Commun., vol. 4, no. 2, pp. 808-819, Mar. 2005.

[31] M. Shayesteh and A. Aghamohammadi, "On the error probability of linearly modulated signals on frequency-flat Ricean, Rayleigh, and AWGN channels," IEEE Trans. Commun., vol. 43, no. 2, pp. 14541466, Feb. 1995.

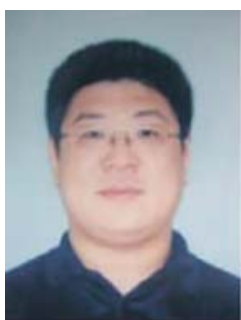

Shuangfeng Han (S'02-M'03) received the B.S. and B.A. degrees in electronics engineering and english for science and technology respectively from Tianjin University in 1995. He worked for Motorola and Huawei company from 1996 to 2000. He received the M.S. and Ph.D. degrees in communication and information systems from Tsinghua University in 2002 and 2006, respectively. He is currently working as a senior engineer for Samsung Electronics telecommunication network R\&D center, Suwon, Korea. His research interests are in the area of distributed wireless and mobile communications

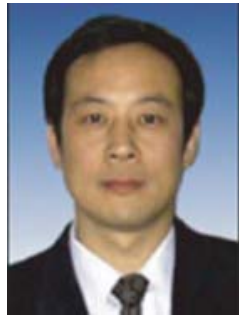

Jing Wang received B.S. and M.S. degrees in electronic engineering from Tsinghua University, Beijing, China, in 1983 and 1986, respectively. He is currently a professor and the vice dean of the School of Information Science and Technology. His research interests are in the area of wireless digital communications. He has published more than 100 conference and journal papers. He is a member of the Technical Group of the China 3G Mobile Communication R\&D Project. He serves as an expert of communication technology in the National 863

Program.

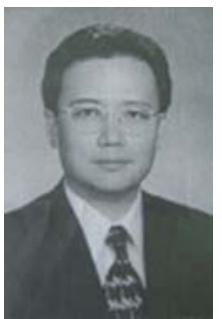

Victor O.K. Li (S'80-M'81-SM'86-F'92) received the SB, SM, EE and ScD degrees in Electrical Engineering and Computer Science from the Massachusetts Institute of Technology, Cambridge, Massachusetts, in 1977, 1979, 1980, and 1981, respectively. He joined the University of Southern California (USC), Los Angeles, California, USA in February 1981, and became Professor of Electrical Engineering and Director of the USC Communication Sciences Institute. Since September 1997 he has been with the University of Hong Kong, Hong Kong, where he is the Chair Professor of Information Engineering at the Department of Electrical and Electronic Engineering. He has also served as Managing Director of Versitech Ltd, the technology transfer and commercial arm of the University, and on various corporate boards. He is a Principal Investigator of the Area of Excellence in Information Technology funded by the Hong Kong government.

Sought by government, industry, and academic organizations, he has lectured and consulted extensively around the world. Prof. Li chaired the Computer Communications Technical Committee of the IEEE Communications Society (1987-1989), and the Los Angeles Chapter of the IEEE Information Theory Group (1983-1985). He co-founded the International Conference on Computer Communications and Networks (IC3N), and chaired its Steering Committee (1992-1997). He also chaired various international workshops and conferences, including, most recently, IEEE INFOCOM 2004 and IEEE HPSR 2005. 
Prof. Li has served as an editor of IEEE NETWORK, IEEE JSAC WIRELESS COMMUNICATIONS SERIES, and Telecommunication Systems. He also guest edited special issues of IEEE JSAC, Computer Networks and ISDN Systems, and the KICS/IEEE JOURNAL OF COMMUNICATIONS AND NETWORKING. He is now serving as an editor of ACM/Springer Wireless Networks and IEEE Communications Surveys and Tutorials.

Prof. Li is a Part-time Member of the Central Policy Unit of the Hong Kong Special Administrative Region (HKSAR). He has been appointed to the Hong Kong Information Infrastructure Advisory Committee by the Chief Executive of HKSAR. He also serves on the Innovation and Technology Fund (Electronics) Vetting Committee, the Small Entrepreneur Research Assistance Programme Committee, the Engineering Panel of the Research Grants Council, and the Task Force for the Hong Kong Academic and Research Network (HARNET) Development Fund of the University Grants Committee. He was a Distinguished Lecturer at the University of California at San Diego, at the National Science Council of Taiwan, and at the California Polytechnic Institute. Prof. Li has also delivered keynote speeches at many international conferences. He has received numerous awards, including, most recently, the Chang Jiang Chair Professorship at Tsinghua University, Beijing, awarded by the Ministry of Education, China, the UK Royal Academy of Engineering Senior Visiting Fellowship in Communications, the Outstanding Researcher Award of the University of Hong Kong, the Croucher Foundation Senior Research Fellowship, and the Bronze Bauhinia Star (BBS), HKSAR, China. He was elected an IEEE Fellow in 1992. He is also a Fellow of the HKIE and the IAE.

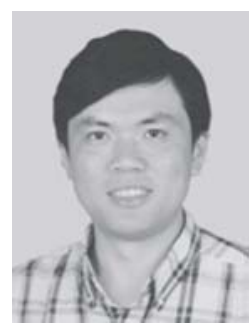

Shidong Zhou (M'98) is a professor at Tsinghua University, China. He received a Ph.D. degree in communication and information systems from Tsinghua University in 1998. His B.S. and M.S. degrees in wireless communications were received from Southeast University, Nanjing, in 1991 and 1994, respectively. From 1999 to 2001 he was in charge of several projects in the China 3G Mobile Communication R\&D Project. He is now a member of the China FuTURE Project. His research interests are in the area of wireless and mobile communica-

tions.

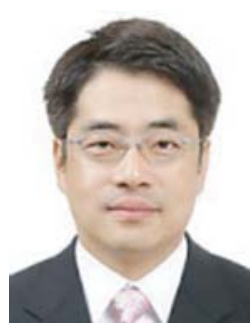

Kyung Park was born in Seoul, Korea, in 1965. He received the B.E and M.E. Degrees in electronic engineering from Yonsei University, Seoul in 1988 and 1990, respectively. From 1990 to 1997, he worked for Agency for Defense Development in Korea where he was engaged in developing HF tactical radio. From 2002 to 2005 he was with the mobile communication research division, Electronics and Telecommunications Research Institute, Korea where he was engaged in developing smart antenna system and 3GPP LCR TDD physical layer technologies. He is currently the leader of the mobile communication standardization team of PANTECH Co. 